\title{
Aligning IS to Organization's Strategy: The INSTAL Method
}

\author{
Laure-Hélène Thevenet ${ }^{1,2}$ and Camille Salinesi ${ }^{1}$ \\ ${ }^{1}$ Université Paris 1 - Panthéon Sorbonne, CRI, 90 rue de Tolbiac \\ 75013 Paris, France \\ ${ }^{2}$ BNP Paribas, Système d'Information Groupe, 41 rue de Valmy \\ 93100 Montreuil Sous Bois, France \\ Laure-Helene.Thevenet@malix.univ-paris1.fr, \\ Camille.Salinesi@univ-paris1.fr
}

\begin{abstract}
Aligning Information Systems (IS) to organization's strategic business objectives is one of organizations' top preoccupations. Misalignment is considered as a reason of IT's failure to improve organizational performance. If strategic alignment is relatively simple to understand, it is not so easy to implement. Our experience showed us that organizations are not really able to systematically evaluate whether there is alignment, mainly because of the lack of documentation on strategic alignment. This paper intends to deal with this issue by proposing an approach to describe organizations' strategic objectives and its IS, in order to document and analyze strategic alignment, i.e. how the IS contributes to strategic objectives satisfaction. The proposed method, called InStAl (Intentional Strategic Alignment), reuses organization documents as a basis to formalize strategic alignment. INSTAL was created following the principles of an action research approach, which consists in developing the approach while exploring issues raised by the case study.
\end{abstract}

Keywords: Alignment, Strategic Alignment Documentation, Organization strategy, Requirements Engineering.

\section{Introduction}

As [1] [2] [3] [4] or [5] already showed, aligning Information Strategy (IS) and business objectives has been considered as a top priority by CIOs and IT executives since several years.

There is a large corpus of empirical and theoretical evidence that alignment improves organizational performance (e.g. [6], [7], [8], [9]). Indeed this latter depends on structures and capabilities that support the successful realizations of strategic decisions. Furthermore, studies highlight the lack of alignment as a major cause for business processes failure in providing return on investments.

Although it is admitted that alignment is impacted by the changing environment, it is still unclear how to achieve and sustain strategic alignment over time. IS and business processes must support the strategy, i.e. the organization's strategic business objectives. Therefore, IS/IT must be deployed to help meeting those objectives. 
However, strategic alignment, i.e. the synergy between the strategic level (organization strategy) and the operational level (BP/IS), must also be controlled and maintained over time despite possible evolutions of IS/IT, of the organization strategy and of the environment.

According to [10], a crucial issue when dealing with strategic alignment lies in the lack of common understanding and communication between the strategy and IS worlds. In fact, actors who define organization strategies, like enterprise executives, (a) do not speak the same language as operational actors (e.g. IS engineers) and (b) do not have the same vision of the organization. The consequence is that IT does not provide the expected value to the organization.

Our method, named INSTAL (INtentional STrategic Alignment), proposes to consider organizations at two levels: (i) the strategic level, which includes the decision-makers' strategy and high level requirements, and (ii) the operational level, which comprises the IS/IT. Based on the observation that documentation usually exists at the two levels while correspondence is seldom systematically documented with the degree of formality needed to support systematic analysis, INSTAL was designed to reuse the two levels' documentation in order to create a third kind of document that describes the synergy between the two levels (i.e. strategic alignment) and the existing links with organization elements such as IT applications, business processes, strategic documentation, etc.

For the organization, the outcomes expected from considering and documenting strategic alignment as a unified view of the strategic and operational levels are: (i) to improve the enterprise agility - its capability to respond to unexpected environmental changes, (ii) to reduce resistance to change, as IS users get a better view on design rationales and on the IS contribution to their own performance, (iii) to improve the visibility of top managers on the IS ROI, in terms of cost savings but also in terms of added value, and on its capacity to answer organization needs, and (iv) to help improve performance evaluation.

On the IS management side, the goals are: (i) to improve the IS flexibility, (ii) to better trace IS evolutions, (iii) to better manage project portfolio, by identifying which IS components are obsolete and redundant and which ones deliver value (needed for arbitration), and (iv) to facilitate impact analysis of evolution requirements.

With these goals in mind, our requirements for a good strategic alignment documentation were to: (i) be formalized using modeling rules, (ii) reflect the complexity of strategic alignment while being able to represent the strategic alignment in a simple manner (using a black box/white box approach), (iii) show alignment as well as mis-alignment (iv) be scalable to real-world organization sizes, (v) deal with strategy and IS on different levels of granularity, and (vi) ensure interoperability with the tools that are already used in organizations.

INSTAL was created using the principles of an action research approach. Basic principles of the method were identified based on bibliography research, then the Seven Eleven Japan (SEJ) case study was explored and the method was constructed each time a new kind of strategic alignment-related issue was encountered.

This paper is structured as follows. Section 2 gives an overview of the INSTAL method, and reports its development with the SEJ case study. Section 3 describes the process model into more detail. Section 4 compares our approach with related works. Our conclusions on this research are given in section 5 . 


\section{Overview of the InStAl Method: Principles, and Alignment Meta-Model Developed with the Case Study}

The INSTAL method is presented in the next section, followed by its development using the SEJ case study and the resulting meta model.

\subsection{The InStAL Method}

Usually, documents about strategy definition and IT components are already present in the organization. For instance business plans, annual reports, strategic reports, performance indicators and scorecards are part of the strategic documentation. IT components, legacy system, IT functionalities, business process are most often documented by specifications. These documents can easily be reused and referenced. However, whereas organization strategies are defined in terms of goals, actors and performance indicators, systems are specified using concepts such as objects, events and functions. This conceptual mismatch results in a difficulty to draw links between them.

One way to obviate this issue is to express both the strategic and operational levels in terms of requirements and with the same language. This approach, recommended by the TOGAF in the context of Enterprise Architecture, allows expressing alignment in a straightforward manner. A review of the RE literature shows that goal-centred languages seem to be the most adequate for this purpose, as they explicitly capture the why and how of both system functionality and organization businesses [11] [12] [13]. Goal modeling has several advantages: goals subsume different concepts such as systems functionalities, business processes or organizational objectives, goals can be refined and therefore be defined at different levels, they can handle the scalability by abstraction mechanisms, and they can be considered as ambivalent as they can integrate different perspectives.

As Fig. 1 shows, the basic principle of the INSTAL method stands in documenting strategic alignment by (i) using a goal-oriented model representing both strategic and operational levels and (ii) defining links between this model and the existing organization's strategic and operational components. Indeed documenting strategic alignment can lead to a network of links between multiple artifacts. For this reason, we use MAPs as an intermediate formalism rather than linking elements directly: they provide a unified and purposeful view on strategic alignment. MAPs sections can be used as a starting point to focus on one particular aspect of alignment based on the purpose we want to deal with. The resulting maps, called Strategic Alignment Maps (SAMs), capture the organization strategy through formalization and the operational level through abstraction. Contribution links are defined between organization's elements and the SAMs and between organization's operational elements and the SAMs. This approach was chosen because we observed in previous experiences that using the MAP formalism to represent both the IS level and the Business Process level is an efficient way to deal with complex problems at multiple levels of granularity and under multiple perspectives ([13]).

Several questions were addressed while exploring the case study: (i) is the MAP technique adapted? Are extensions needed?, (ii) is it well-suited to model both 


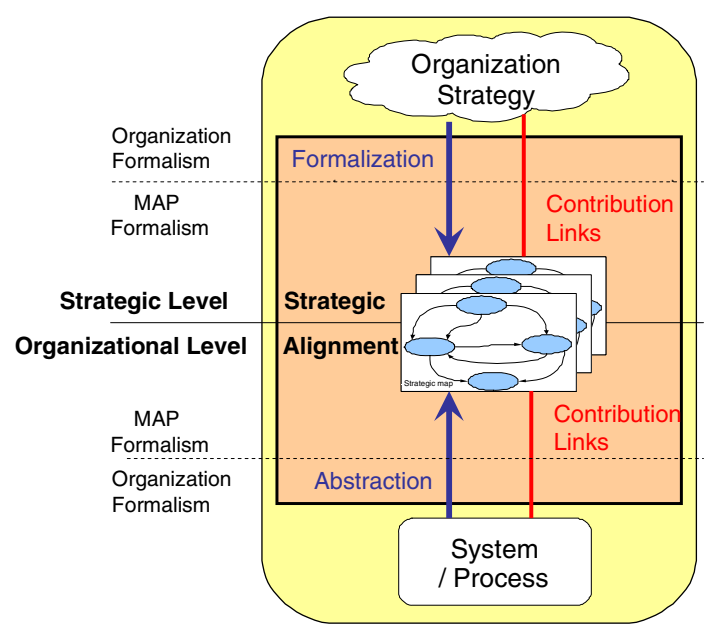

Fig. 1. Overview of the InStAL method

organization strategy (non functional items) and functional items in the same model?, (iii) what are the types of links needed between SAMs and existing organization's elements?, and (iv) what is the process to apply the InSTAL method?

\subsection{Description of the SEJ Case Study}

The SEJ case study was already well described in the literature (e.g. [14]). This particular case study was chosen to develop INSTAL because it is a real case study (and not a toy example), a large amount of data is available, and more importantly, it had already been used to evaluate another strategic alignment approach [15] [16].

SEJ is the largest chain in the Japanese convenience retailing industry. The enterprise has franchise contracts with local shops all over Japan and supplies exclusive products and services to franchisees. The distribution centers, that distribute these products and services to shops, are also independent proprietors.

The SEJ supply chain is complex and implies several partners like suppliers, distributors, logistics providers, and franchise stores.

SEJ's major asset is information rather than physical properties. Indeed, SEJ's strategy is to use information to meet customer's demands, so that they can always find "what they need when they need it" in SEJ franchised stores. Coupled with an effective delivery service, this strategy helps in increasing sales, lowering the number of unsold items, and reducing the need for storage space, which is important in Japan where space is rare.

Having the right product at the right time calls for gathering very diverse information: purchasing habits, the store's neighborhood from both social and environmental perspectives, weather, local events, etc. All this data is analyzed in real-time in order to forecast what the customer might need at the exact time they shall need it. 
Several information sources are available for the strategy and the IS. We particularly used annual reports ${ }^{1}$. The analysis of these documents allowed identifying the following strategic objectives: (i) Get better value of SEJ stores by answering to any client's needs ("To meet customer needs, products must be delivered just in time and only when needed"), (ii) Live in harmony with the local communities and (iii) Respect the environment.

As defined earlier, a primary concern was to create a unified model of these strategic objectives that would also provide a view on SEJ's IS. This task was undertaken using the MAP formalism, which was adapted at the same time to achieve the actual goal of documenting strategic alignment.

\subsection{The MAP Formalism}

A map is an oriented graph where nodes are goals (or intentions) and edges are strategies. A map is composed of sections that contribute to achieving a high level goal. A section is a triplet $<G_{i}, G_{j}, S_{i j}>$ and represents a way to achieve the target goal $G_{j}$ following the strategy $S_{i}$ taking into account that the goal $G_{i}$ should have already been achieved. Maps organize goals and strategies to represent a flow of decisions.

Indeed, a section has for source a goal when its achievement is a precondition to undertake the strategy. The other way round, as soon as a goal is achieved, any section that starts from it can be undertaken at anytime.

Each map has two special goals, Start and Stop. The Start goal corresponds to the entry point of the process. Sections containing this goal can always be undertaken. The Stop intention allows to specify sections that aim at completing the goal described by the map. A more detailed definition of MAP can be found in [17].

\subsection{Documenting Strategic Alignment Using MAP}

Fig. 2 shows an example of two SAMs that were described to document the strategic objective "Get better value of SEJ stores by answering to any client's needs" and part of the IS that supports it. The study of SEJ values and aspirations (visibility, availability towards customers, innovation, anticipation etc), made emerge two main goals in the high-level SAM: Ensure the Control of resources (such as time, space, stores, products) and Increase sources of value (such as customers, products quality and organization efficiency). These two goals are ambivalent, they can represent the organization strategy, but also tackle the operational level. Besides the strategies attached to these goals can be refined. Therefore, goals can be defined at different levels of abstraction and a collection of goals can be structured in a goal graph.

These goals can be attained in different ways (strategies in MAP formalism). The $M_{1}$ map describes sections composed of goals and strategies. The latter details how SEJ can attain its strategic goals in accordance with its organization strategy. For example, one way to increase sources of value is by having the lowest prices, but as we can see with strategies between start and (c) Increase the sources of value, this option has not been retained by SEJ. SEJ chose to develop quality products/services and to have the right products rather than having only basic products at a lower price.

\footnotetext{
${ }^{1}$ SEJ website: http://www.sej.co.jp/english/
} 


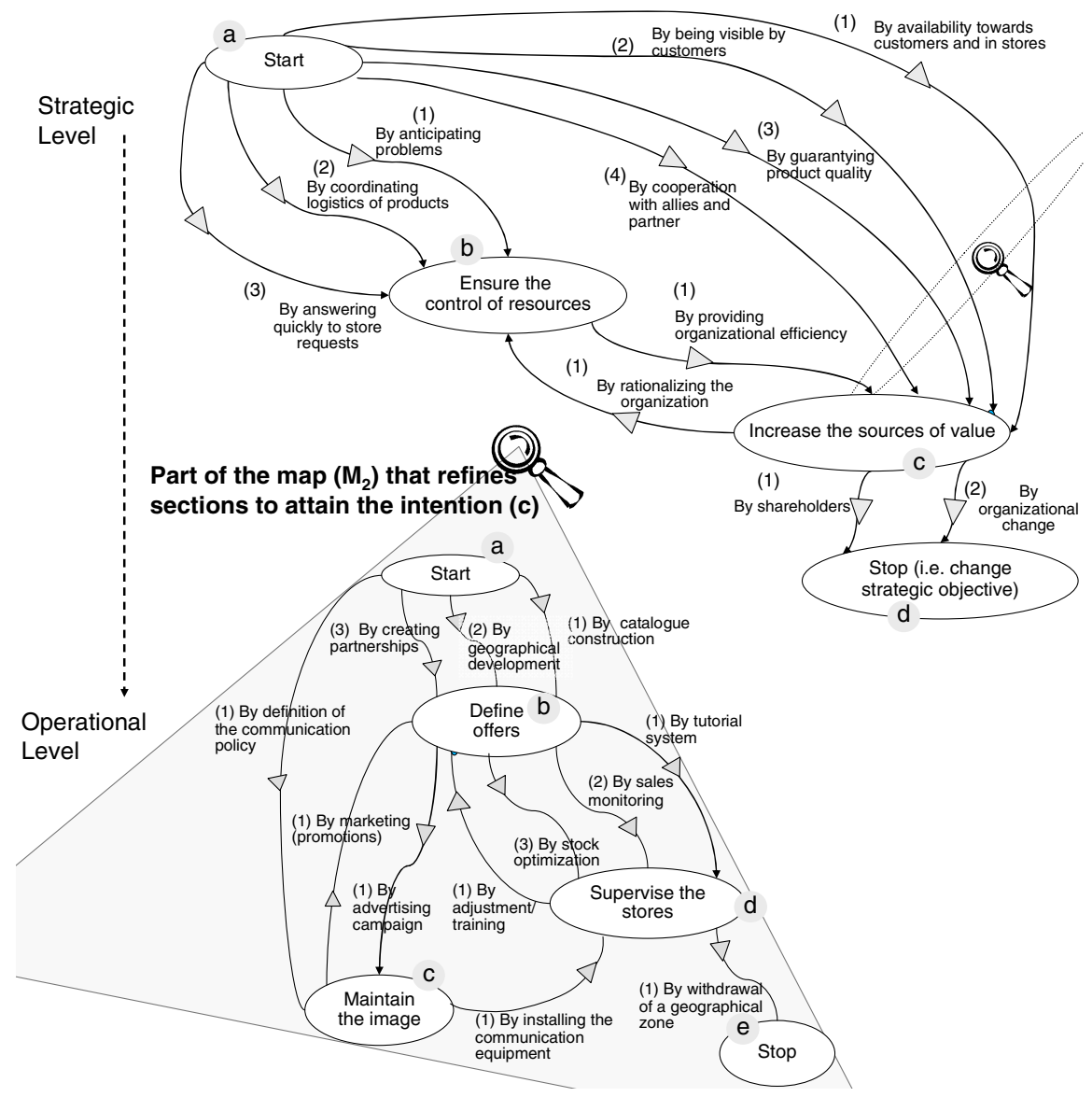

Fig. 2. Part of Strategic alignment maps representing the strategic objective: "Get better value of SEJ stores by answering any client's needs"

The second map $\mathrm{M}_{2}$, describes, using a black box / white box approach, details on how SEJ addresses the strategic goal (c) Increase the sources of value. Goals present in $\mathrm{M}_{2}$ are: "Define Offers", "Supervise the stores" and "Maintain the image". The goal "Define Offers" can be attained by three strategies that detail what SEJ must do to offer product and services in accordance with the organization strategy (have the right product at the right time, have products/services of quality etc.) which includes: (i) to define the catalogue of products and services (adapted to the customers' needs), (ii) to develop partnerships with suppliers, organizations (e.g. to allow clients to pay electricity bills), transport companies etc., and (iii) to develop its sites: stores and warehouses. These strategies contribute to increase the sources of value (customers, sales etc.) by availability (in catalogue, in stores), by visibility (network of stores), and by developing quality (products in catalogue and logistics for fresh products).

We designed the $\mathrm{M}_{2}$ map by focusing on goals that are important to SEJ without dealing with their operational details. For example, the goal "Define offers" presents 
an important goal for SEJ that relies on the operational goal "define catalogue", which can appear in the MAP refining the strategy ab1.

Developing the maps presented in Fig. 2 revealed that it is possible to represent both the strategic and operational level in a single map with an abstract view, and that the black box/white box approach could be used to deal with complex problems.

However, while the map was developed using strategic documentation and specifications of IS components, the links between all these documents were still not explicit and even sometimes unclear. For example, sections of the high-level map $\mathbf{M}_{1}$ can be linked to some strategic documents like business plans, annual reports and internal documents. The set of SAMs describe the AS IS based on strategic documentation and IS components and the AS Wished that can for example highlights manual processes or processes to redesign. The refined map $\mathbf{M}_{2}$ is, by construction, mostly related to IS components. For example the section $<$ Start, Define offers, By catalogue construction > was identified by analyzing IS features. If we refine this section, we can see systems (i) that analyze customers' profiles from questionnaires about their marital status, activities when visiting stores, their proximity with the store in POS (Point of sale) and others (ii) to analyze purchases hour by hour and product by product. These clearly correspond to items to include in strategic alignment documentation as they are needed to anticipate sales, to optimize store storage and so to contribute to having the right product at the right time.

While creating the SAMs, we were lead to reason about the links between strategy and the IS, and discovered that there are a number of different links and that these links can be combined in complex constructions. The different types of link discovered during this analysis are presented in the next section.

\subsection{Resulting Alignment Meta Model}

Fig. 3 presents the product meta model associated to the INSTAL method using the UML formalism. As the meta model shows, strategic alignment is documented by contribution links defined between the strategic alignment map (SAM) and the strategic components on the one hand, and operational components on the other hand.

The SAM meta model reuses the basic concepts of the MAP formalism except for the refinement mechanism which is handled differently. Indeed, in SAMs, several sections can be refined in one SAM, while in the traditional MAP meta model, refinement is a one-to-one relationship between a section and a finer grain map.

As shown in Fig. 3, contribution links are defined between an element and one or several strategic or operational components. An element is either a SAM section or a formula, a formula being defined as a set of SAM sections separated by logic $A N D / O R$ operators. For example, (ab1 AND ab2) is a formula composed of two members (in this case two SAM sections) separated by the AND operator. Components refer to any organization documents: strategic components are for example business plans or annual reports. Operational components can be application specifications, models (e.g. UML use case diagrams) or business processes. Links are oriented from the components to the SAM. They can also be defined in the other direction. For example, specifying that a component "is necessary to" achieve a section of a SAM is equivalent to specifying that the SAM section "requires" the component. 


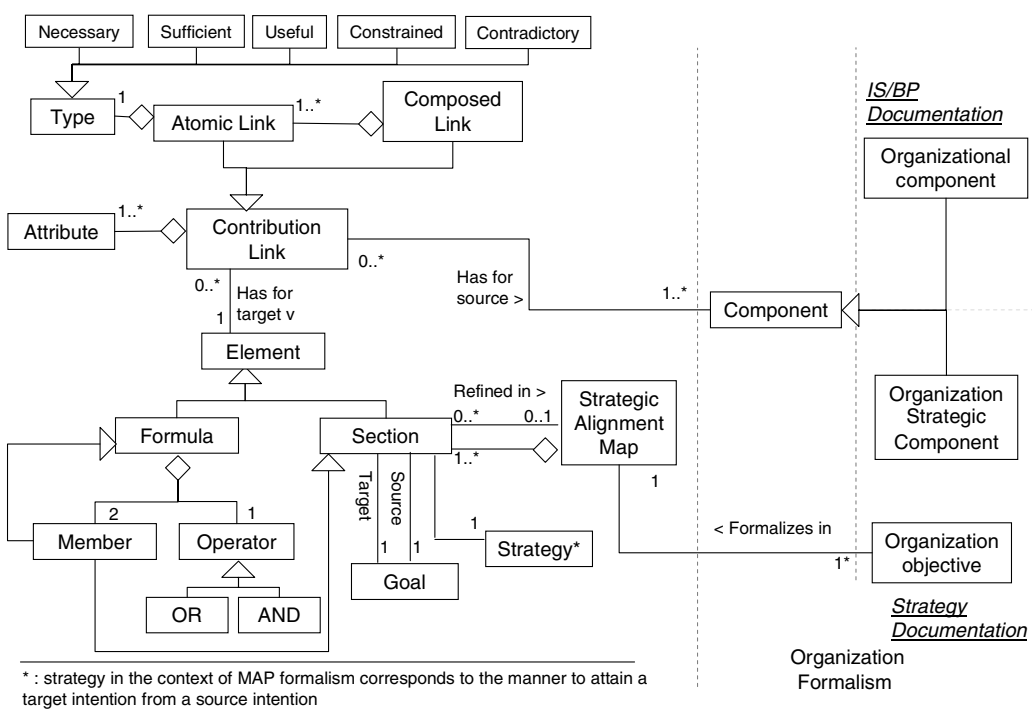

Fig. 3. Strategic Alignment Meta model

Five types of atomic links were identified while exploring the SEJ case study: "is necessary to", "is sufficient to", "is useful to", "is constrained by" and "is contradictory with". Contribution links can also be composed. The valid composition of contribution link types are: "necessary and sufficient", "useful and sufficient", "useful and constrained by". As the definition of contribution links is not an easy task, quite subjective, attributes related to contribution links have been defined in the meta model as a date of last revision; percentage of trust and associated metrics/measures.

Let $\mathrm{C}$ be a component (strategic or operational) and $\mathrm{M}$ an element of a SAM, the atomic links defined between $\mathrm{C}$ and $\mathrm{M}$ are defined as follows.

Is Necessary To: $\mathrm{C}$ is necessary to $\mathrm{M}$ (equivalent to $\mathrm{M}$ requires $\mathrm{C}$ ) means that the fulfillment of a map element $\mathrm{M}$ cannot occur if that of the strategic or operational component $\mathrm{C}$ is not performed.

If evolution impacts $\mathrm{C}$ or $\mathrm{M}$, it is inevitable to verify that the link is preserved, and if not, that the choice is intentional. If $\mathrm{M}$ or $\mathrm{C}$ evolves, either the necessary link is preserved or it is modified, the latter implying the update of the link type and the verification that there is still one or more links of necessary type going to $\mathrm{M}$. In the SEJ case study, the strategic document that describes the SEJ's objective to answer any clients' needs is necessary to the section ab1: <Start, Define offers, by catalogue construction $>$. In the same way, the three IT applications that allow managing catalogue $(\mathrm{OC} 1)$, having the suppliers catalogue $(\mathrm{OC} 2)$ and having the result of sales by different criteria (OC3) (e.g. by products, by stores, by geographical zone) in real time are necessary and sufficient to the section ab1. It means that $\mathrm{OC} 1$ is necessary to $\mathrm{M}$, OC2 is necessary to $\mathrm{M}$, OC3 is necessary to $\mathrm{M}$ and (OC1 and OC2 and OC3) are necessary and sufficient to $\mathrm{M}$. If OC3 evolves and is no more able to provide sales report in real-time, the system regresses for this functionality. So either it is a choice to be less reactive but to have others gains (e.g. more cross information, reports), or it 
is not and so the link between OC3 and $\mathrm{M}$ is no more of type necessary, and the set of OC1, OC2 and OC3 is no more necessary and sufficient to $\mathrm{M}$.

Is Useful To: $\mathrm{C}$ is useful to $\mathrm{M}$ (equivalent to $\mathrm{M}$ draws part of $\mathrm{C}$ ) indicates a dependency of a weaker nature than the "is necessary to" link. This link specifies that the strategic or operational component $\mathrm{C}$ helps in realizing/satisfying the element $\mathrm{M}$, but is not in any way mandatory.

In the case study, the applications that allow managing the best practices catalogue, reporting past problems and giving an overview of sales and purchases by annual period are useful to the section ab1: <Start, Control the resources, By anticipating problems $>$ because they help supervising the shops, but they are not sufficient. For example, the analysis of some data (e.g. sales by shop, sales by product, and sales by geographical zone) is necessary to better help and advice stores.

If several components are useful to an element, it means that these components can be complimentary or alternative. It is interesting to study them to examine if there is some redundancy in the IS. For example, the best practice management and the past problem report might be in the same system, in particular if these two systems are used by the same users and if there is some possible consolidation. It might have an application that proposes best practices with some of them based on real problems that occurred in the past.

Is Sufficient To: $\mathrm{C}$ is sufficient to $\mathrm{M}$ (equivalent to $\mathrm{M}$ satisfies by $\mathrm{C}$ ) means that realizing the strategic or operational component $\mathrm{C}$ is enough to satisfy the fulfillment of the element M. The study of the other types of links (necessary and useful) allows to highlight some redundant and obsolete systems which don't provide added value and so that should be replaced or deleted.

Is Constrained By: $\mathrm{C}$ is constrained by $\mathrm{M}$ (equivalent to $\mathrm{M}$ constraints $\mathrm{C}$ ) (e.g. the realization of $\mathrm{M}$ is influenced or limited by those of $\mathrm{C}$ ). This type of link means that the strategic or operational components have more capabilities than what they are referenced for in the SAM. It is interesting to analyze these types of link to either update the SAM to integrate these opportunities, or to document this link with a justification. For example, an ERP can have more possible functionalities than the ones implemented in the organization.

Is Contradictory With: $\mathrm{C}$ is contradictory with $\mathrm{M}$ (equivalent to $\mathrm{M}$ excludes the realization of $\mathrm{C}$ in a certain context) indicates that a strategic or operational component $\mathrm{C}$ can be contradictory with an element. This link puts in perspective the cases where alignment is not assured or outlines conflicting decisions.

\section{The InStAl Process Model}

Fig. 4 provides an overview of the process that was used to apply the INSTAL method in the SEJ case study. The process model is formalized with the traditional MAP formalism so as to focus on methodological goals and supports to achieve these goals rather than on process details such as sequences of activities.

Three methodological goals are addressed by the method: (b) Identify Strategic/ Operational Items, (c) Construct a SAM and (d) Define link between section and Strategic/Operational components. 
(1) By abstracting IS components

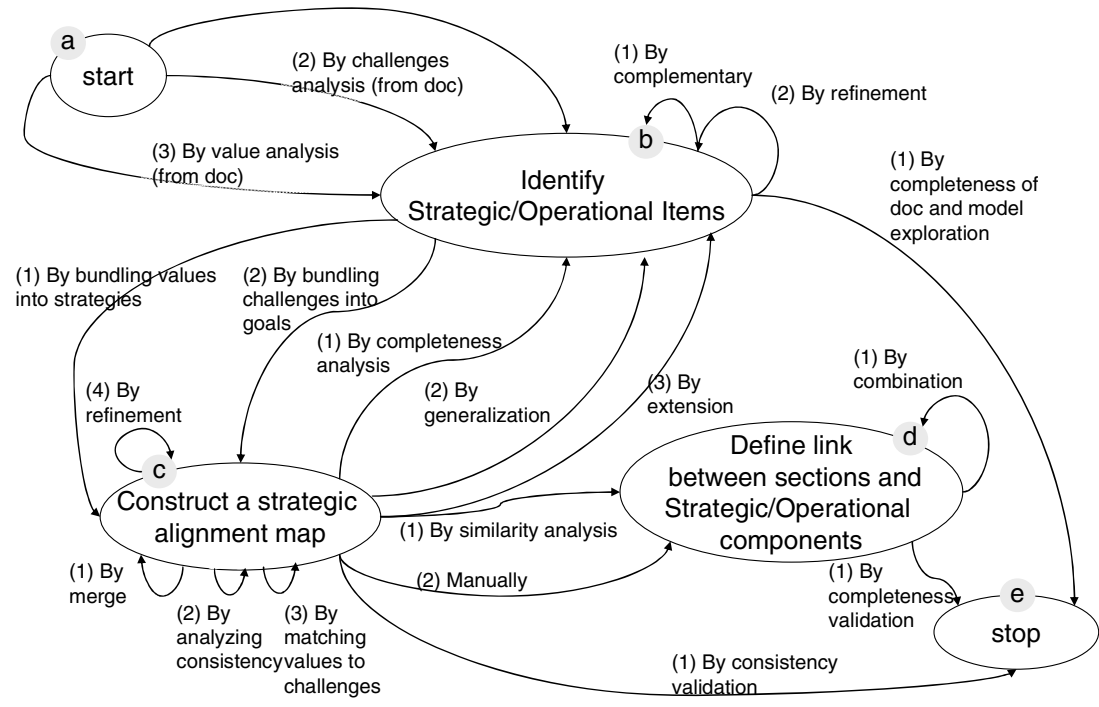

Fig. 4. Process model to document strategic alignment

"Items" is the general term used to address strategic and operational items. They are identified from documents and interviews and contribute to define the sections of SAMs. Once items are identified, they are used to define the SAMs.

Once the SAMs are specified, it is possible to link elements (i.e. SAM sections or formula) with strategic or operational components already present in the organization. The SEJ experience revealed that several strategies could be used to identify items, construct a SAM, define contribution links and terminate the process.

\subsection{InStAL Strategies to Achieve the Goal (b) Identify Strategic/Operational Items}

The goal (b) Identify Strategic/Operational Items can be achieved through three strategies originating from (a) Start: (1) by abstracting IS components, (2) by challenges analysis from strategic documents and (3) by value analysis from strategic documents, and from two reflexive strategies (1) by complementarity and (2) by refinement. As the SAM is ambivalent, the map definition is based on both the operational and strategic elements. In the strategy (1) the IS components are abstracted to have high level functionalities, in strategies (2) and (3) strategic documents are analyzed to find organization's challenges and values. Challenges are finally expressed as elements that should not be lost to increase benefits. In the SEJ case study, examples of challenges are: clients, space, stores, market share, time, organization's quality, suppliers, products quality. Values are high level qualities chosen by the organization to address the challenges. Examples of values in the SEJ case study are: availability, visibility, products' quality, coordination, speed, rationality, and anticipation.

The two reflexive strategies allow finding complementary and more detailed items. 


\subsection{InStAL Strategies to Achieve the Goal (c) Construct a Strategic Alignment Map}

The strategic goal (c) Construct a strategic alignment map can be attained from the source goal (b) Identify Strategic/Operational Items by two strategies (1) By bundling values into strategies and (2) By bundling challenges into goals. Two categories of challenges were defined in the SEJ case study: (i) challenges related to resources, and (ii) challenges related to the sources of values that should be increased. The former leads to the strategic goal "ensure the control of the resources" (time, space, stores, products and services). The latter leads to the goal "increase the sources of values" (market share, client, products' and services' quality, and organization's quality). Several strategies to reach these goals were specified by studying SEJ values. The strategic goal "Increase the sources of value" can be attained by four strategies. These are based on different values such as availability, visibility, quality, coordination and cooperation (see sections ac1, ac2, ac3 and ac4 in Fig. 2).

Four recursive strategies on the goal (c) Construct a strategic alignment map, are proposed: (1) by merge, (2) by analyzing consistency, (3) by matching values to challenges and (4) by refinement. These strategies allow to improve a SAM or to define other SAM by refinement of section(s).

From a SAM it is possible to identify strategic and operational items (1) by completeness analysis and (2) by refinement. For example, the section ab1 (Fig. 2) in $\mathrm{M}_{2}:<$ Start, Define offers, By construction catalogue $>$ can be refined in an other map to describe how SEJ defines offers, for example by analyzing the customers' needs from sales, local events, weather forecasting etc. New items might be identified to construct the refined map.

\subsection{InStAl Strategies to Achieve the Goal (d) Define Link Between Sections and Strategic/Operational Components and to Stop the Process}

Once all items have been identified and used to define the set of SAMs, it is possible to stop the process (1) by completeness of documentation and model exploration. Besides, further exploration of the case study revealed several different kinds of links between strategic or operational components, and SAM's section which have been defined previously. The strategies used to achieve this task are (1) by similarity analysis and (2) manually. Searching in components' documentation about a part of a goal or a strategy can help finding the automatic link between a section and a component. Manual link means interviewing the person in charge of the component (e.g. top-managers, functional architect) or using documentation to define the appropriate type(s) of link and define a new link.

The recursive strategy (1) by combination allows to define formula (several sections with OR/AND links) and a link between this set of sections and components.

It is possible to stop the process from the goals: (b) Identify Strategic/operational Items (1) by completeness of documentation and model exploration, (c) Construct a strategic alignment map (1) by consistency validation and (d) Link items with Strategic/Operational components (1) by completeness validation. 


\section{Related Works}

\subsection{Goal Modeling in RE}

Different approaches have been developed in the Requirements Engineering (RE) community to express high level requirements for IS. The goal modeling approaches allow defining the purpose of the system from an external point of view, in particular from a user perspective. Among the best known methods, there are $i^{*}$ [18], CREWSL'Ecritoire [19], and KAOS [20].

$I^{*}$ is a method that aims at modeling the relationships between actors and their goals. Bleistein et al. [15] [16] have adapted $\mathrm{i}^{*}$ to model both strategic and operational goals. The authors uses $i *$ representation (adapted using the BRG-Model conceptual framework) to represent the strategy then the context diagram from Jackson's Problem Frames to model the IT context of strategy achievement. The approach represents the business strategies and the IS description in the same model, with the same formalism and relates them through simple and mono-typed contribution links. One issue with this approach relates to the $i^{*}$ notation, which does not use the black box/white box strategy and produces complex models when the number of goals increases. Indeed the main difficulties with $i^{*}$ is that it lacks (i) systematic goal refinement mechanisms (all goals are in the same model), and has no (ii) goal-strategy couple to help clarifying the multiple ways in which a goal can be achieved. Besides, our experience with the MAP formalism showed us that MAPs are particularly adapted to dealing with multi purpose systems, which helps managing complex situations, whereas it has been clearly demonstrated that $i^{*}$ models find their limit when the situation gets complex.

CREWS-L'Ecritoire combines goal modeling and scenario analysis to guide the elicitation of user goals. Scenario are not used in the INSTAL method, nevertheless the CREWS-L'Ecritoire uses pre-defined levels that could be reused to better guide the application of the black box / white box paradigm.

In KAOS goal models, goals are linked through AND/OR decomposition links. This allows refining high-level goals into finer grain goals down to concrete system requirements. Refinement cannot be separated from OR decomposition and AND decomposition which introduces artificial complexity in the goal hierarchy, while the main issue is to sort goals according to their level of abstraction and relate goals when they belong to the same level of abstraction.

As Bleistein et al show [15][16], although some aspects of the organization strategy can be taken into account with these goal models, none of them (except for Bleistein's adaptation of $\left.i^{*}\right)$ really addresses the issue of strategic alignment.

\subsection{Research on IS Alignment}

Different kinds of concepts are involved in strategic alignment depending on authors. A well known model is Henderson's and Venkatraman's Strategic Alignment Model [21] that inspired numerous models such as [22] [23] [24]. The Strategic Alignment Model defines the interrelationship between business strategies and IT strategies in a clear way. However, it does not provide a practical framework to document strategic alignment. Luftman [22] and Maes [23] define alignment between organization 
strategy and IT people. However, they do not propose a method for designing the strategy. Other authors focus on alignment of software architecture and business process architecture [25], [26], or on alignment between system and business processes [27] [28] [29] [30]. These approaches concern the improvement of alignment. However, alignment is not considered as a concept by itself that can be documented and therefore used to support systematic reasoning.

Salinesi [31] showed that a systematic method should use a notation to document and reason on IS alignment. This view is also supported by EA approaches [32] [33].

Notations for documenting alignment are considered in [31] [34] [35] and [36], but not at the strategic level, which is different by nature from the operational level.

\section{Conclusions}

This paper has described the INSTAL method for IS strategic alignment, which was developed using the principles of an action research approach that consists in exploring the SEJ case study. The paper has shown that (i) MAP formalism is adapted to document models both at the strategic and operational levels, and that (ii) complex links can be defined to document organization's strategic and operational components involvement in strategic alignment. A process model was also presented.

The experience revealed some limitations in our approach:

- Only research documents, articles and SEJ's annual reports were used in the case study. No SEJ internal documents or interviews were used, which could be considered as a bias of the case study with respect to real world situation.

- Our exploration of the SEJ case study did not consider the entire company. This is a bias towards validating the scalability of the approach. Besides, different kinds of problems might have arisen if the study had been complete.

- No quantitative evaluation is proposed. Our only validation stands in the SEJ case study which was addressed as an empirical and subjective experiment.

- We did not use a tool to systematically check the degree of completeness and consistency of the documentation of strategic alignment.

Our current works concern the development of a technique to analyze SAMs and contribution links with the aim of improving strategic alignment. We also believe that a more complete validation should be undertaken using empirical evaluations, and interviews of experts to explore the usability of the SAMs and the effectiveness of the INSTAL method. Last, our experience at BNP-Paribas and our participation in industrial workgroups showed us that organizations face different alignment-related problems such as: difficulty to maintain legacy systems that would be replaced if sufficient benefit had justified the high cost of change, difficulty for top managers to acquire visibility on the IS results and on strategic alignment to determine priorities for investment etc. To meet these needs, we think that it is essential to explore further extension of the INSTAL method in the following directions:

- Facilitate traceability between high-level requirements and IS components in order to support impact analysis and to systematically examine how new projects contribute to the organization strategy and to the strategic alignment. 
- Support IS redundancy analysis: IS can be huge and lack good documentation, so it happens that two systems that have similar functionalities are developed in two different departments, which generates double costs. We believe the analysis of strategic alignment documentation could highlight this kind of redundancy and either avoid new developments or at least trace its rationale.

- Measures are essential to analyze strategic alignment and answer the concrete preoccupations of top managers, it would be interesting to study the possibility of associating measures and metrics to contribution links.

- Document To-Be SAMs and support comparative analysis between the AS-IS and To-Be SAMs so as to develop a better vision of what must be addressed in the target and the path to reach it (using contribution links). Any project could show how it contributes to the target strategic alignment.

\section{References}

1. Luftman, J., Maclean, E.R.: Key issues for IT executives. MIS Quarterly Executive 4(2), 89-104 (2004)

2. Reich, B.H., Nelson, K.M.: In Their Own Words: CIO Visions About the Future of InHouse IT Organizations. The DATA BASE for Advances in ISs 34, 28-44 (2003)

3. Tallon, P.P., Kraemer, K.L.: Executives' Perspectives on IT: Unraveling the Link between Business Strategy, Management Practices and IT Business Value. ACIS2002, USA (2002)

4. Watson, R.T., Kelly, G.G., Galliers, R.D., Brancheau, J.: Key issues in information systems management: an international perspective. Journal of Management ISs 13, 91-115 (1997)

5. Brancheau, J.C, Janz, B., Wetherbe, J.C.: Key issues in information systems management. 1994-95. SIM Delphi results. MIS Quarterly 20, n(2), 225-242 (1996)

6. Chan, Y.E., Huff, S.L., Copeland, D.G., Barclay, D.W.: Business Strategic Orientation, Information Systems Strategic Orientation and Strategic Alignment. Information Systems Research 8(2), 125-150 (1997)

7. Kefi, H., Kalika, M.: Survey of Strategic Alignment Impacts on Organizational Performance in Int. European Companies, Hawaii Int. Conf. on System Sciences (2005)

8. Chan, Y.E.: Why haven't we mastered alignment? The importance of the informal organization structure, MIS Quarterly Executive 1, 97-112 (2002)

9. Croteau, A.M., Bergeron, F.: An information technology trilogy: business strategy, technological deployment and organizational performance. Journal of Strategic Information Systems 10, 77-99 (2001)

10. Luftman, J.: Assessing Business-IT Alignment Maturity. Communications of the Association for Information Systems 4, $\mathrm{n}^{\circ}(14), 1-50$ (2000)

11. Yu, E.: Agent Orientation as a Modelling Paradigm. Wirtschaftsinformatik. Vol.43 (2) (2001)

12. Van Lamsweerde, A.: Goal-Oriented Requirements Engineering: A Guided Tour, Invited Paper, Int. Symposium on Requirements Engineering (RE), pp.249-263 Toronto, Canada (2001)

13. Rolland, C., Salinesi, C.: Modeling Goals and Reasoning with Them, Engineering and Managing Software Requirements (EMSR), Aurum, A., Wohlin, C. Springer Verlag (2005)

14. Bensaou, M.: Seven-Eleven Japan: Managing a Networked Organization, INSEAD EuroAsia Centre, Case Study (1997) 
15. Bleistein, S., Aurum, A., Cox, K., Ray, P.: Strategy-Oriented Alignment in Requirements Engineering: Linking Business Strategy to Requirements of e-Business Systems using the SOARE approach. Journal of Research and Practice in IT 36, 259-276 (2004)

16. Bleistein, S., Cox, K., Verner, J., Phalp, K.: B-SCP: a requirements analysis framework for validating strategic alignment of organisational IT based on strategy, context and process. Information and Software Technology 46, 846-868 (2006)

17. Rolland, C., Prakash, N.: Matching ERP System Functionality to Customer Requirements. RE01, Canada pp. 66-75 (2001)

18. Yu, E.: Towards Modelling and Reasoning Support for Early-Phase Requirements Engineering. RE97 Washington D.C., USA pp. 226-235 (1997)

19. Rolland, C., Souveyet, C., Ben Achour, C.: Guiding goal modelling using scenarios. IEEE Transactions on Software Engineering vol. 24(12) (1998)

20. Dardenne, A., Lamsweerde, A., Fickas, S.: Goal-directed Requirements Acquisition. Science of Computer Programming 20, 3-50 (1993)

21. Henderson, J.C., Venkatraman, N.: Strategic alignment: Leveraging information technology for transforming organizations. IBM Systems Journal 32(1), 4-16 (1993)

22. Luftman, J.N.: Competing in the Information Age. Oxford University Press, Oxford (1996)

23. Maes, R.: A Generic Framework for Information Management. Prime Vera Working Paper, Universiteit Van Amsterdam (1999)

24. Goedvolk, H., van Schijndel, A., van Swede, V., Tolido, R.: The Design, Development and Deployment of ICT Systems in the 21st Century: Integrated Architecture Framework (IAF). Cap Gemini Ernst and Young (2000)

25. Aerts, A.T.M., Goossenaerts, J.B.M., Hammer, D.K., Wortmann, J.C.: Architectures in context: On the evolution of business, application software, and ICT platform architectures. Information and Management 41, $\mathrm{N}^{\circ}(6), 781-794$ (2004)

26. Wieringa, R.J., Blanken, H.M., Fokkinga, M.M., Grefen, P.W.P.J.: Aligning application architecture to the business context, CAiSE03, Austria, pp.209-225 (2003)

27. Bodhuin, T., Esposito, R., Pacelli, C., Tortorella, M.: Impact Analysis for Supporting the Co-Evolution of Business Processes and Supporting Software Systems. BPMDS04, Latvia

28. Arsanjani, A., Alpigini, J.: Using Grammar-oriented Object Design to Seamlessly Map Business Models to Component-based Software Architectures. Int. Symposium of Modelling and Simulation, USA, pp.186-191 (2001)

29. Giaglis, G.M.: On the Integrated Design and Evaluation of Business Processes and Information Systems. Communications of the AIS, Vol 2, N`5, July (1999)

30. Kardasis, P., Loucopoulos, P.: Aligning Legacy Information Systems to Business Processes, CAiSE98, Italy, pp. 25-40 (1998)

31. Salinesi, C., Rolland, C.: Fitting Business Models to Systems Functionality Exploring the Fitness Relationship. CAiSE03, Austria (2003)

32. Zachman, J.A.: A framework for Information Systems architecture. IBM Systems Journal 26, $\mathrm{N}^{\circ}(3), 276-292$ (1987)

33. Longépé, C.: The Enterprise Architecture IT Project: The Urbanisation Paradigm. Elsevier Health Sciences, New York (2005)

34. Etien, A., Rolland, C.: A Process for Generating Fitness Measures, CAISE05, pp. 277 292. Springer, Portugal (2005)

35. Wegmann, A., Regev, R., Loison, B.: Business and IT Alignment with SEAM, REBNITA, RE05, France (2005)

36. Soffer, P.: Fit Measurement: How to Distinguish Between Fit and Misfit, note for BPMDS'04, Latvia (2004 